\title{
Consolidation of the postural set during voluntary intermittent light finger contact as a function of hand dominance
}

\author{
David Kaulmann ${ }^{1}$, Hui-Ya Chen ${ }^{2}$, Leif Johannsen ${ }^{1,3}$ \\ ${ }^{1}$ Human Movement Science, Department of Sport and Health Sciences, Technische Universität München, Germany \\ ${ }^{2}$ School of Physical Therapy, Chung Shan Medical University, Taichung, Taiwan \\ ${ }^{3}$ School of Health Sciences, Faculty of Medicine and Health Sciences, University of East Anglia, Norwich, United Kingdom
}

\begin{abstract}
Light fingertip contact with an earth-fixed referent decreases body sway. In a previous study Johannsen et al. (2014) demonstrated longer return-to-baseline of body sway for intermittent contacts of more than 2 seconds duration. This indicates that sway reduction with light tactile contact involves postural control strategies independent of the availability of tactile feedback and may depend on the intention to control body sway with light touch feedback. In the present study, we investigated the effect of hand dominance on post-contact return-to-baseline to probe for potential inter-hemispheric differences in the utilization of light finger contact for sway control. Twelve healthy, right-handed young adults stood in normal bipedal stance with eyes closed on a force plate with an earth-fixed referent directly in front. Acoustic signals instructed onset and removal of intermittent light touch. We found that return-to-baseline of sway following longer contact durations is affected by hand dominance with the dominant hand resulting in a slower return to No-contact levels of sway. Our results indicate that the light touch postural set is more persistent and might need longer to disengage when established with the dominant hand or takes longer to consolidate when established with the non-dominant hand.
\end{abstract}

\section{INTRODUCTION}

In daily life, we often establish intermittent haptic contact with objects in our environment to orientate ourselves and to yield stability of body balance. For example, walking down the aisle on a moving train carriage, we move from handhold to handhold prepared to counter any unexpected perturbations. Or when we cross an unlighted room, we haptically move from contact to contact to gain an estimate of our position and to augment our sense of spatial orientation.

Light fingertip contact with an earth-fixed reference leads to a reduction in body sway [1]. Only a few studies have addressed the time course of sway before and after a contact transition [2, 3, 4]. Sway stabilization with light touch is a time-consuming integrative and attention demanding process $[2,3,5]$.

In terms of a multimodal sensory strategy, it seems rather costly if the postural control system switches between different multisensory sets each time intermittent contact is established or removed [6]. Instead, while anticipating upcoming contact intervals and thus the imminent availability of reliable haptic feedback, keeping a multisensory set including the haptic channel temporarily active might offer an advantage with respect to the costs of switching the postural sets [7]. For example, Bove and colleagues (2006) demonstrated that the intention to establish contact within less than 5 seconds leads to reductions in body sway before contact is established. Schiepatti and colleagues [8] proposed that transient anticipatory processes are involved in the preparation of the central postural set to the context of stance control with light contact. Investigating intermittent touch with only short contact durations, Johannsen et al. (2014) demonstrated that contact durations of more than $2 \mathrm{~s}$ result in slower recovery of reduced sway to baseline levels after contact removal. These observations indicate that the integration of fingertip contact requires no less than about 2 seconds and is likely to involve not only bottom-up sensory processing but also top-down, "intentional" control of body sway and tactile attention.

The two hemispheres of the human brain might play different roles in the control of body sway with and without light touch $[9,10]$. In the present study we not only aimed to replicate previous findings with intermittent but longer contact durations, we also intended to probe for differences between the dominant and non-dominant hemispheres regarding their influence on switching the postural set in right-handed participants during phases of intermittent light touch.

\section{METHODS}

Participants

Twelve healthy young adults (mean age $=25.8, \mathrm{SD}=2.6 ; 7$ woman and 5 men) were recruited for the current study. Inclusion criteria were (1) right hand dominance and (2) no balance impairment. All participants were informed about the study protocol and signed a written informed consent was provided. The study was approved by the Clinical Research Ethics committee of the Technical University of Munich.

\section{Procedure}

Participants stood barefoot in normal bipedal stance. After the height of the stand was adjusted to each participant's waist level, participants were asked to hold their index finger of the dominant hand above a touch plate while keeping the outstretched arm in a comfortable posture. We instructed participants to close their eyes, and to stand relaxed but as still as possible without speaking.

Trials were started when participants indicated that they were ready. On hearing a high-pitched tone, participants flexed their index finger at the metacarpal-phalangeal joint to initiate light finger contact. On a low-pitched tone, participants lifted their index finger just above the touch plate. Before testing participants could practice the task in order to 
familiarize themselves with the experimental protocol. Afterwards they performed at least 6 trials with $30 \mathrm{~s}$ break in between hands.

After participants finished sway testing, we assessed the tactile discrimination threshold of each hand's index fingertip using 13 orientation gratings with a gap width ranging from $0.35 \mathrm{~mm}$ to $5.50 \mathrm{~mm}$ [11]. Participants had to judge whether gratings were aligned straight or orthogonal with the fingertip. Gratings were applied manually for about two seconds. Testing protocol consisted of a staircase procedure which ended either after ten successful reversals or a total of 50 grating presentations. The final tactile acuity threshold was derived from the average of the last 10 presentations.

\section{Apparatus}

A force plate $(600 \mathrm{~Hz}$; Bertec FP4060-10, USA) measured the six components of the ground reaction forces and moments to determine the antero-posterior $\left(\mathrm{COP}_{\mathrm{ap}}\right)$ and medio-lateral $\left(\mathrm{COP}_{\mathrm{ml}}\right)$ components of Centre-of-Pressure. In response to a high-pitched or low-pitched auditory cue, participants either made or withdrew fingertip contact with a touch plate ( $3 \mathrm{~cm}$ diameter), mounted on a stand at waist level to the front of the participants. A force-torque transducer (ATI Nano17, USA) measured the normal and horizontal shear forces applied to the touch plate with a rate of $200 \mathrm{~Hz}$. We measured body kinematics ( $60 \mathrm{~Hz}$; Zebris, Germany) in terms of trunk motion with three acoustic markers placed at wrist, shoulder and hip.

Each balance testing consisted of 2 blocks of at least 6 trials per hand (range $=6$ to 8 trials; blocked, randomized order: dominant hand, non-dominant hand). Every balance trial contained four auditorily triggered active transitions between No-touch and Touch ("onset") and Touch and No-touch ("removal"). The acoustically cued intermittent active contact durations were $1 \mathrm{~s}, 1.5 \mathrm{~s}, 10 \mathrm{~s}$ and $20 \mathrm{~s}$ in randomized order. Every No-contact interval was at least 10 seconds long. Onset and removal time points were randomized resulting in total trial durations of at least $130 \mathrm{~s}$.

\section{Data reduction and statistical analysis}

All data were interpolated to $600 \mathrm{~Hz}$ and merged before low-pass filtering with a fourth-order Butterworth filter (10 $\mathrm{Hz}$ cut-off frequency) and differentiated to yield rate of change. According to the vertical touch force as detected by the force-torque sensor, onset and removal time points of each touch period were determined. For comparisons between contact durations participants' actual contact durations were sorted into the following categories: T1 $(0.8 \mathrm{~s}-1.6 \mathrm{~s}), \mathrm{T} 2(2.0$ $\mathrm{s}-2.6 \mathrm{~s}), \mathrm{T} 10(8.0 \mathrm{~s}-13.0 \mathrm{~s})$ and T20 (18.0 s-22.0 s). Trial segments with other contact durations were discarded. Subsequently, the T1 and T2 categories were averaged and subsumed under "short" duration conditions, while T10 and T20 were averaged and combined as "long" contact durations for statistical analysis.

Non-discarded trial segments were divided into bins of $500 \mathrm{~ms}$ duration from $5 \mathrm{~s}$ before to $5 \mathrm{~s}$ after a contact transition. Sway within each bin was quantified in terms of the standard deviation (SD) of the Centre-of-Pressure velocity in the anterior-posterior $\left(\mathrm{dCOP}_{\mathrm{ap}}\right)$ direction. Sway parameters were averaged for each duration condition of all trials a participant performed.

Using SPSS 18.0 software (Chicago, IL, USA), repeated-measures ANOVAs were performed with time course across a range of $500 \mathrm{~ms}$ bins, contact duration and contacting hand as within-subject factors.

In order to characterise the return of sway to the Nocontact baseline following contact removal, we fitted linear regressions across three time bins: $0.5 \mathrm{~s}$ before removal, $0.5 \mathrm{~s}$ and $1 \mathrm{~s}$ after removal. Statistical analysis of regression slope and zero-offset was conducted with repeated-measures ANOVAs with contact duration and contacting hand as within-subject factors. Level of significance was set to $p=.05$ after Greenhouse-Geisser correction. Effects with estimated effects sizes of partial $\eta^{2}>0.14$ were considered large.

\section{RESULTS}

Statistical analysis of the tactile discrimination thresholds revealed no significant differences between the dominant and non-dominant hands $(\mathrm{p}=0.33)$, which suggests that hand dominance did not influence tactile sensitivity of the respective hand. Figure 1 shows the tactile sensitivity thresholds for the index finger of both hands.

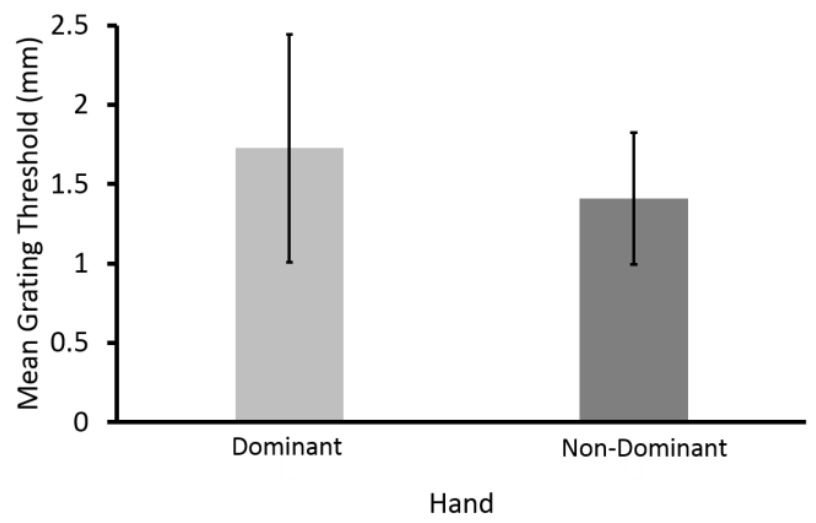

Figure 1. Tactile sensitivity threshold in terms of the just noticeable gap width for the dominant (light grey) and nondominant (dark grey) hand. Error bars indicate standard error of the mean.

Figure 2 shows average sway progression from $5 \mathrm{~s}$ before to $5 \mathrm{~s}$ after contact onset and Figure 3 shows average sway progression around contact removal for short (upper panel) and long (lower panel) contact durations. Sway is oscillating close to the No-contact baseline before contact is established. After the onset of touch, sway transiently rises above and then begins to drop below the baseline. Similarly, sway with light touch is noticeably below the baseline before contact is removed. Following contact removal, sway once again overshoots the No-contact baseline and then settles towards it. 

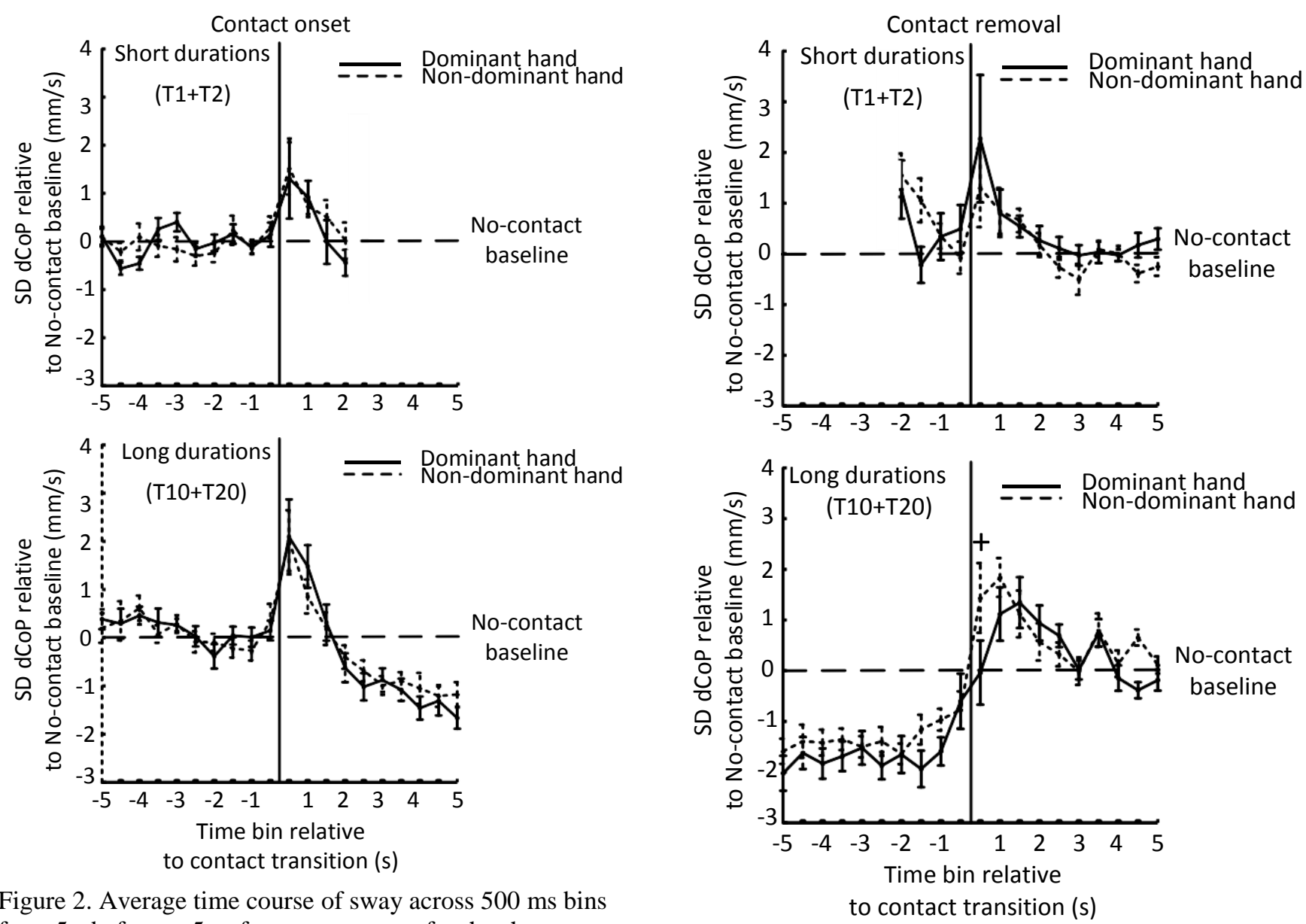

Figure 2. Average time course of sway across $500 \mathrm{~ms}$ bins from $5 \mathrm{~s}$ before to $5 \mathrm{~s}$ after contact onset for the short durations (upper panel) and long durations (lower panel) for the dominant (bold line) and non-dominant hand (dashed line). Error bars indicate standard error of the mean.

Although steady-state sway with light touch of the dominant hand (time bins from $5 \mathrm{~s}$ to $.5 \mathrm{~s}$ before contact removal) appears lower compared to the non-dominant hand, the two contact conditions were statistically not different $\left(\mathrm{p}>.25\right.$, partial $\left.\eta^{2}=.12\right)$.

The increase in sway after removal of long duration light touch appears less rapid with the dominant hand compared to the non-dominant hand. In order to assess the return-tobaseline of sway after contact removal (including the overshoot), we examined the time course of sway during the removal transitions. Focussing on the range from 0.5 seconds before to 1.5 seconds after. We found statistical significant interactions of between hand and contact duration $\left(\mathrm{F}(1,11)=6.83, \mathrm{p}=.02\right.$, partial $\left.\eta^{2}=.38\right)$ as well as between hand, contact duration and time course $(\mathrm{F}(3,33)=4.18, \mathrm{p}=.03$, partial $\left.\eta^{2}=.28\right)$. Post-hoc single comparisons showed a strong difference between the dominant and non-dominant hand at the $0.5 \mathrm{~s}$ time bin after long duration contact removal $\left(\mathrm{F}(1,11)=3.47, \mathrm{p}=.08\right.$, partial $\left.\eta^{2}=.24\right)$ with lower sway after contact removal of dominant hand.

Figure 3. Average time course of sway across $500 \mathrm{~ms}$ bins from $5 \mathrm{~s}$ before to $5 \mathrm{~s}$ after contact removal for the short durations (upper panel) and long durations (lower panel) for the dominant (bold line) and non-dominant hand (dashed line). Error bars indicate standard error of the mean. The cross indicated the tendency of a difference between both hands $(\mathrm{p}>.1)$.

Sway overshoot after removal of the non-dominant hand had progressed further during this period, almost reaching peak overshoot, compared to the dominant hand. Peak overshoot, although numerically lower following contact with the dominant hand, was not affected by limb dominance (....).

Analysis of the linear regression parameters showed significant interactions between contact durations and hand for the regression slope $\left(F(1,11)=6.89, p=.02\right.$, partial $\left.\eta^{2}=.39\right)$ and offset $\left(F(1,11)=6.70, p=.03\right.$, partial $\left.\eta^{2}=.38\right)$. For both slope and offset after short duration contact, post-hoc single comparisons did not show differences between hands. After long duration contact, however, previous contact with the dominant hand resulted in a lower slope $(\mathrm{F}(1,11)=5.55, \mathrm{p}=.04$, partial $\left.\eta^{2}=.34\right)$ and offset $(\mathrm{F}(1,11)=4.81, \mathrm{p}=.05$, partial $\eta^{2}=.30$ ) compared to the non-dominant hand. Figure 4 shows linear regression slope and offset of the sway progression following contact removal for short and long contact durations as a function of the hand tested. 



Contact durations

Figure 4. Linear regression slope (upper panel) and offset (lower panel) for short and long contact durations for the dominant (light grey bars) and non-dominant (dark grey bars) hand. Error bars indicate standard error of the mean. An asterisk indicates a significant comparison between hands $(\mathrm{p}<0.05)$.

\section{DISCUSSION}

Actively removing intermittent light touch at the fingertip leads to a rapid increase in sway within $500 \mathrm{~ms}$ after contact removal for contact durations shorter than 2.5 seconds irrespective of the contacting hand. Similarly, contact at the fingertip of the non-dominant hand also shows rapid increase for longer durations. In contrast, more persistent contact with the dominant hand results in delayed sway return-to-baseline.

In our present study, the general progression of sway during a contact removal transition is in line with the previous study of Johannsen et al. [4]. They showed that short contact durations initiate a reduction in sway but do not result in a significant reduction. A delayed return-to-baseline only occurred for contact durations longer than 2 seconds. Contact durations longer than 5 seconds, however, were not tested. Therefore, our present study tested longer contact durations, which ought to more likely result in steady-state sway with light contact. Indeed, we found that the sway progression after touch removal increased at a lower rate but only when longer duration touch was established with the dominant hand. With the nondominant hand, contact resulted in a rapid sway increase similar to the short contactdurations.

A central question to be answered is whether the less rapid, more gradual return of sway to No-contact levels after removal of the dominant hand resembles a functional advantage or disadvantage? It could be that a rapid return expresses a fast readjustment in the multisensory strategy of the postural control system. The instantiation of a new postural set involving the haptic channel could result in inter-sensory conflict between an information-deprived haptic channel and the other senses. The sway overshoot observed could be a consequence of the sudden deprivation of a highly weighted tactile signal leading to acute intermodal conflict. For example, following abrupt cessation of long-term support surface sway referencing, Peterka and Loughlin demonstrated the emergence of transient, involuntary $1 \mathrm{~Hz}$ body oscillations, possibly due to overcorrective torque production [12].

It seems more reasonable to delay postural set switching until the likelihood is high that the haptic channel will provide reliable feedback for an extended period. Once such a steady state has been reached it also seems reasonable to keep this set active and delay disengagement, if further contact periods are expected to occur in the near future. This reasoning seems to apply to the pattern we observed for the dominant hand. As we tested right-handed participants it implies that the dominant left hemisphere is involved in this strategy. In a previous study, we observed that disruption of the left-hemisphere inferior parietal gyrus (IPG) by repetitive transcranial magnetic stimulation

(rTMS) inhibited sway overshoot following unexpected, passive removal of light contact [4]. This could mean that the left IPG plays a role in the detection of multisensory conflict or the directing of tactile attention. This is in correspondence with reports by Ishigaki and colleagues [13], who suspected involvement of the left primary somatosensory and posterior parietal cortices in the processing and integration of steadystate right hand light touch. On the other hand, we disrupted the left and right PPC by cTBS and did not find anyalterations in sway progression following removal of active light touch [10].

Nevertheless, all-in-all the evidence suggests that the lefthemisphere plays some role in the control of body sway with

light haptic feedback form the contralateral, right hand, for example in the consolidation of an adequate central postural set.

Why did the non-dominant, left hand not demonstrate a delayed return-to-baseline similar to the dominant, right hand? One possibility is that consolidation of the central postural set for the light touch with the non-dominant hand has a longer time constant. For example, our participants might have been more used to explore the environment with their dominant hand.

An aftereffect on postural sway following an extended duration of lightly gripping a cane was reported by Oshita and Yano [14]. They investigated the effect of lightly touching a cane on postural sway and ankle- joint muscle activity. They found decreased sway and decreased co-contraction of the ankle joint muscles when the cane was gripped lightly. These reductions were also present after lifting off the cane from the ground. In interestingly, their participants used the left hand to grip the cane, presumably the non-dominant hand. Oshita and Yano did not assess varying contact durations but $30 \mathrm{~s}$ contact only. It seems that also light contact with the non-dominant hand can lead to slow return-to-baseline of body sway. Perhaps contact durations of more than $20 \mathrm{~s}$ duration are the 
prerequisite.

To conclude, the occurrence of a delayed return-to-baseline of sway following removal of fingertip light touch is affected by hemispheric lateralization. While the dominant hand showed a delayed return-to-baseline effect after long contact durations, it was not observed when the non-dominant hand was used for contact. This difference cannot be explained by differences in the tactile sensitivity of the contacting index fingers of the two hands. Instead, the effect could rely on a difference in the rate of consolidation of a light touch postural set, with faster consolidation when tactile feedback is processed in the dominanthemisphere.

\section{ACKNOWLEDGMENTS}

We acknowledge the financial support by the Federal Ministry of Education and Research of Germany (BMBF; 01EO1401) and by the Deutsche Forschungsgemeinschaft (DFG) through the TUM International Graduate School of Science and Engineering (IGSSE).

\section{REFERENCES}

[1] Holden M, Ventura J, Lackner JR. Stabilization of posture by precision contact of the index finger. J Vestib Res, 1994. 4:285-301

[2] Rabin, E., P. DiZio, and J.R. Lackner, Time course of haptic stabilization of posture. Experimental Brain Research, 2006. 170(1): p. $122-126$.

[3] Sozzi, S., et al., Sensorimotor integration during stance: processing time of active or passive addition or withdrawal of visual or haptic information. Neuroscience, 2012. 212: p. 59-76.

[4] Johannsen, L., et al., Disruption of contralateral inferior parietal cortex by $1 \mathrm{~Hz}$ repetitive TMS modulates body sway following unpredictable removal of sway-related fingertio feedback. Neuroscience Letters, 2014. 586:13-8.

[5] Vuillerme, N., Isableu, B., Nougier, V., Attentional demands associated with the use of a light fingertip touch for postural control during quiet standing, 2006. 169(2):232-6.

[6] Prochazka, A., Sensorimotor gain control: a basic strategy of motor systems. Progress in Neurobiology, 1989, 33: 281-307.

[7] Monsell, s., Task switching. Trends Cogn Sci, 2003. 7(3):134-140.

[8] Bove, M., et al., The postural disorientation induced by neck muscle vibration subsides on lightly touching a stationary surface or aiming at it.Neuroscience, 2006. 143(4):1095-103.

[9] Yadav, V. \& Sainburg, R.L., Handedness can be explained by a serial hybrid control scheme. Neuroscience, 2014. 278:385-96.

[10] Kaulmann, D., Hermsdörfer, J., Johannsen, L., Disruption of right Posterior Parietal Cortex by cTBS alters the control of body balance in quiet stance. Eur J Neuroscience, 2017.

[11] Van Boven, RW. \& Johnson, KO. The limit of tactile spatial resolution in humans: grating orientation discrimination at the lip, tongue, and finger. Neurol, 1994. 44:2361-2366.

[12] Peterka, R.J., Loughlin, P.J., Dynamic regulation of sensorimotor integration in human postural control. J Neurophysiology, 2004. 91(1):410-23.

[13] Ishigaki, T., et al., EEG frequency analysis of cortical brain activities induced by effect of light touch. Exp Brain Res, 2016. 234(6):142940.

[14] Oshita, K. \& Yano, S. Effect and immediate after-effect of lightly gripping the cane on postural sway. Journal of Physiological Anthropology, 2016. 35:14. 